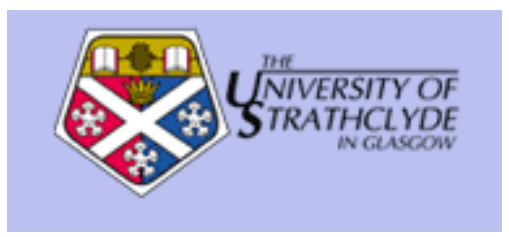

Sullivan, J.M. and Wilson, S.K. and Duffy, B.R. (2008) Air-blown rivulet flow of a perfectly wetting fluid on an inclined substrate. In: Progress in Industrial Mathematics at ECMI 2006. Springer, pp. 774-778. ISBN 978-3-540-71991-5

http://strathprints.strath.ac.uk/15078/

This is an author produced version of a paper published in Progress in Industrial Mathematics at ECMI 2006. Springer, pp. 774-778. ISBN 978-3-540-71991-5. This version has been peer-reviewed but does not include the final publisher proof corrections, published layout or pagination.

Strathprints is designed to allow users to access the research output of the University of Strathclyde. Copyright (C) and Moral Rights for the papers on this site are retained by the individual authors and/or other copyright owners. You may not engage in further distribution of the material for any profitmaking activities or any commercial gain. You may freely distribute both the url (http://strathprints.strath.ac.uk) and the content of this paper for research or study, educational, or not-for-profit purposes without prior permission or charge. You may freely distribute the url (http://strathprints.strath.ac.uk) of the Strathprints website.

Any correspondence concerning this service should be sent to The Strathprints Administrator: eprints@cis.strath.ac.uk 


\title{
Air-Blown Rivulet Flow of a Perfectly Wetting Fluid on an Inclined Substrate
}

\author{
Julie M. Sullivan, Stephen K. Wilson and Brian R. Duffy
}

Department of Mathematics, University of Strathclyde, Livingstone Tower, 26 Richmond Street, Glasgow G1 1XH, United Kingdom

\section{Introduction}

Thin-film flows occur in a variety of physical contexts including, for example, industry, biology and nature, and have been the subject of considerable theoretical research. (See, for example, the review by Oron, Davis and Bankoff [4].) In particular, there are several practically important situations in which an external airflow has a significant effect on the behaviour of a film of fluid, and consequently there has been considerable theoretical and numerical work done to try to understand better the various flows that can occur. (See, for example, the studies by King and Tuck [2] and Villegas-Díaz, Power and Riley [6].) The flow of a rivulet on a planar substrate subject to a shear stress at its free surface has been investigated by several authors, notably Myers, Liang and Wetton [3], Saber and El-Genk [5], and Wilson and Duffy [9]. All of these works concern a non-perfectly wetting fluid; the flow of a rivulet of a perfectly wetting fluid in the absence of a shear stress at its free surface has been treated by Alekseenko, Geshev and Kuibin [1], and by Wilson and Duffy $[7,8]$. In the present short paper we use the lubrication approximation to obtain a complete description of the steady unidirectional flow of a thin rivulet of a perfectly wetting fluid on an inclined substrate subject to a prescribed uniform longitudinal shear stress at its free surface.

\section{Problem Formulation}

Consider the steady unidirectional flow of a thin rivulet with constant semiwidth $a$ and constant volume flux $Q$ of a perfectly wetting fluid subject to a prescribed uniform longitudinal shear stress $\tau$ at its free surface on a planar substrate inclined at an angle $\alpha$ to the horizontal. Cartesian axes $O x y z$ are chosen with the $x$-axis down the slope, the $y$-axis parallel to the substrate $z=0$, and the $z$-axis normal to the substrate. The fluid is assumed to be Newtonian with constant density $\rho$, viscosity $\mu$, and surface tension $\gamma$. The 
velocity $\mathbf{u}=u(y, z) \mathbf{i}$ and pressure $p=p(x, y, z)$ of the fluid are governed by the familiar mass-conservation and Navier-Stokes equations subject to the usual normal and tangential stress balances and the kinematic condition at the free surface $z=h(y)$, and no slip at the substrate $z=0$. Since the fluid is perfectly wetting the contact angle is zero at the contact lines $y= \pm a$ (where, by definition, the rivulet has zero thickness).

We consider a thin rivulet with a small transverse aspect ratio $\epsilon \ll 1$; in this case it is appropriate to non-dimensionalise $y$ and $a$ with $l, z$ and $h$ with $\epsilon l, u$ with $U=\rho g \epsilon^{2} l^{2} / \mu, Q$ with $\epsilon l^{2} U=\rho g \epsilon^{3} l^{4} / \mu, p-p_{\infty}$ and $\tau$ with $\rho g \epsilon l$, where $l=(\gamma / \rho g)^{1 / 2}$ is the capillary length, $g$ is acceleration due to gravity, and $p_{\infty}$ is the uniform atmospheric pressure.

Since the flow is unidirectional, the mass-conservation equation and kinematic boundary condition are satisfied identically, and at leading order in $\epsilon$ the Navier-Stokes equation reduces to

$$
0=\sin \alpha+u_{z z}, \quad 0=-p_{y}, \quad 0=-p_{z}-\cos \alpha,
$$

which can readily be solved subject to boundary conditions of no slip at the substrate, $u=0$ on $z=0$, balances of normal and tangential stress at the free surface, $p=-h^{\prime \prime}$ and $u_{z}=\tau$ on $z=h$, and appropriate conditions at the contact lines, $h=0$ and $h^{\prime}=0$ at $y= \pm a$, where the prime denotes differentiation with respect to argument, to give the solution

$$
u=\frac{\sin \alpha}{2}(2 h-z) z+\tau z, \quad p=(h-z) \cos \alpha-h^{\prime \prime} .
$$

Substituting the solution for $p$ into the second equation in (1) yields a thirdorder ordinary differential equation for the free surface profile $h$, namely $\left(h^{\prime \prime}-\right.$ $\cos \alpha h)^{\prime}=0$ to be solved subject to (??). This elementary problem was solved by Wilson and Duffy [7] who showed that there is no solution for $h$ when $0 \leq \alpha \leq \pi / 2$ (i.e. no solution corresponding to a sessile rivulet or a rivulet on a vertical substrate), but that there is a solution when $\pi / 2<\alpha \leq \pi$ (corresponding to a pendent rivulet), namely

$$
a=\frac{\pi}{m}, \quad h=\frac{h_{\mathrm{m}}}{2}(1+\cos m y),
$$

where $m=\sqrt{|\cos \alpha|}$ and $h_{\mathrm{m}}=h(0)$ is the maximum height of the rivulet. The volume flux down the rivulet $Q$ is given by

$$
Q=\int_{-a}^{+a} \int_{0}^{h} u \mathrm{~d} z \mathrm{~d} y=\frac{\pi}{24 m}\left(5 \sin \alpha h_{\mathrm{m}}+9 \tau\right) h_{\mathrm{m}}^{2} .
$$

If the flux takes the prescribed value $Q=\bar{Q}$, then (4) determines the appropriate value(s) of $h_{\mathrm{m}}$. Once $h_{\mathrm{m}}$ is known the rivulet solution given by (2) and (3) is completely determined. In the special case of no prescribed shear stress, $\tau=0$, and in the limit of large prescribed shear stress, $|\tau| \rightarrow \infty$, we obtain the simple explicit solutions 


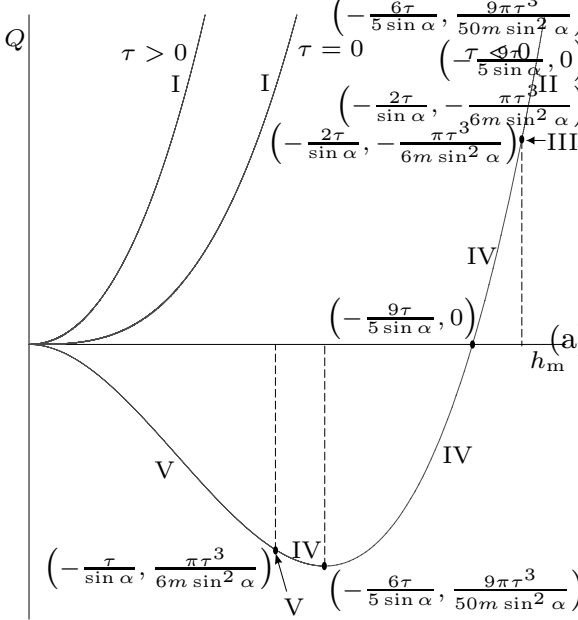

Fig. 1. Sketch of $Q$ as a function of $h_{\mathrm{m}}$ for $\tau>0, \tau=0$ and $\tau<0$, showing when the different types of flow pattern occur.
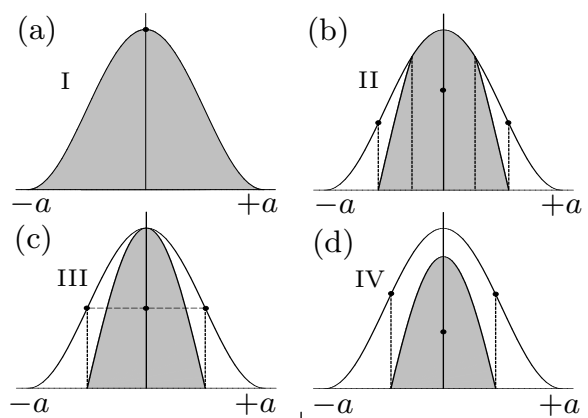

(d)

(e)

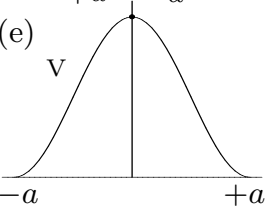

Fig. 2. Sketch of the five different types of flow pattern. Regions of downward flow are shaded and regions of upward flow are unshaded.

$$
h_{\mathrm{m}}=\left(\frac{24 m \bar{Q}}{5 \pi \sin \alpha}\right)^{\frac{1}{3}} \quad \text { and } \quad h_{\mathrm{m}}=\left(\frac{8 m \bar{Q}}{3 \pi \tau}\right)^{\frac{1}{2}}
$$

respectively.

\section{Rivulet Solutions}

Figure 1 shows a sketch of $Q$ given by (4) as a function of $h_{\mathrm{m}}$ for $\tau>0, \tau=0$ and $\tau<0$. For $\tau \geq 0, Q$ is a monotonically increasing function of $h_{\mathrm{m}}$ tending to infinity as $h_{\mathrm{m}} \rightarrow \infty$. In contrast, for $\tau<0, Q$ initially decreases monotonically to a minimum value $Q=Q_{\min }$, where $Q_{\min }=9 \pi \tau^{3} / 50 \mathrm{~m} \sin ^{2} \alpha(<0)$, at $h_{\mathrm{m}}=$ $h_{\text {min }}=-6 \tau / 5 \sin \alpha$, before increasing monotonically through the value $Q=0$ at $h_{\mathrm{m}}=h_{\mathrm{m} 0}$, where $h_{\mathrm{m} 0}=-9 \tau / 5 \sin \alpha$, and eventually tending to infinity as $h_{\mathrm{m}} \rightarrow \infty$. The number of solutions for $h_{\mathrm{m}}$ thus depends on the sign of $\tau$ and the value of $\bar{Q}$. When $\tau \geq 0$, there is one solution when $\bar{Q}>0$, but there are no solutions when $\bar{Q} \leq 0$. When $\tau<0$, there is one solution when $\bar{Q} \geq 0$ with $h_{\mathrm{m}} \geq h_{\mathrm{m} 0}$ and there are two solutions when $Q_{\min }<\bar{Q}<0$, a "thin" solution with $0<h_{\mathrm{m}}<h_{\min }$ and a "thick" solution with $h_{\min }<h_{\mathrm{m}}<h_{\mathrm{m} 0}$; when $\bar{Q}=Q_{\min }$ there is a single solution $h_{\mathrm{m}}=h_{\mathrm{min}}$, and when $\bar{Q}<Q_{\min }$ there are no solutions. 


\section{Classification of Flow Patterns}

Figure 2 shows a sketch of the five different types of cross-sectional flow patterns that can occur; regions of downward flow (i.e. $u>0$ ) are shaded and regions of upward flow (i.e. $u<0$ ) are unshaded. When $\tau>0$ the prescribed shear stress acts down the substrate in cooperation with the effect of gravity. As a result, the flow is downward throughout the rivulet (we refer to this flow pattern as type I; see Figure 2(a)). When $\tau<0$, the prescribed shear stress acts up the substrate in opposition to the effect of gravity, which leads to more interesting behaviour than in the case $\tau \geq 0$. In particular, we find that although the velocity can be downward within the rivulet, it is always upward near the contact lines. When $h_{\mathrm{m}}>h_{\mathrm{III}}=-2 \tau / \sin \alpha$ there is both upward and downward flow on the free surface (type II, Figure 2(b)). When $h_{\mathrm{m}}=h_{\mathrm{III}}$ the flow is upward on the free surface except at $y=0$ and $z=h_{\mathrm{m}}$, where the velocity is zero (type III, Figure 2(c)). When $h_{\mathrm{V}}<h_{\mathrm{m}}<h_{\mathrm{III}}$, where $h_{\mathrm{V}}=-\tau / \sin \alpha$, the flow is always upward on the free surface (type IV, Figure 2(d)) but is downward within part of the rivulet. Finally, when $h_{\mathrm{m}} \leq h_{\mathrm{V}}$ the effect of the prescribed shear stress dominates that of gravity and the flow is upward throughout the rivulet (type V, Figure 2(e)). Figure 1 summarises when the different types of flow pattern occur.

\section{Solutions for Prescribed $\tau$ and Varying $\alpha$}

Figure 3 shows a plot of $h_{\mathrm{m}}$ as a function of $\alpha / \pi$ when $\tau=1$ and is typical of all such plots for $\tau>0$. When $\bar{Q}>0$ there is a single solution for $h_{\mathrm{m}}$ for all $\pi / 2<\alpha \leq \pi$ and all solutions are of type I. Figure 4 shows a plot of $h_{\mathrm{m}}$ as a function of $\alpha / \pi$ when $\tau=-1$ and is typical of all such plots for $\tau<0$. When $\bar{Q} \geq 0$ there is a single solution for $h_{\mathrm{m}}\left(\geq h_{\mathrm{m} 0}\right)$ for all $\pi / 2<\alpha \leq \pi$. However, when $\bar{Q}<0$ there can be no, one or two solution(s) for $h_{\mathrm{m}}\left(<h_{\mathrm{m} 0}\right)$. When $Q_{\mathrm{c}}<\bar{Q}<0$, where $Q_{\mathrm{c}}=9 \pi 5^{1 / 4} \tau^{3} / 40 \simeq 1.0570 \tau^{3}(<0)$, there is a thick and a thin solution for all $\pi / 2<\alpha \leq \pi$. When $\bar{Q}=Q_{\mathrm{c}}$ these solutions coincide at $\alpha=\alpha_{\mathrm{c}}=\pi-\tan ^{-1} 2 \simeq 0.6476 \pi$ and $h_{\mathrm{m}}=h_{\mathrm{mc}}=h_{\min }\left(\alpha_{\mathrm{c}}\right)=-3 \tau / \sqrt{5} \simeq$ $-1.3416 \tau$, while for $\bar{Q}<Q_{\mathrm{c}}$ there are two disconnected branches of solutions, each consisting of a thick and a thin solution which coincide on the curve $h_{\mathrm{m}}=h_{\mathrm{min}}$. Figure 4 also shows how the curves $h_{\mathrm{m}}=h_{\mathrm{III}}$ and $h_{\mathrm{m}}=h_{\mathrm{V}}$ divide the $\alpha-h_{\mathrm{m}}$ plane into regions in which different types of flow pattern occur.

\section{Conclusions}

We have obtained a complete description of the steady unidirectional flow of a thin rivulet of a perfectly wetting fluid on an inclined substrate subject to a prescribed uniform longitudinal shear stress at its free surface. In ongoing work we are analysing the stability of such a rivulet to small perturbations and investigating when it is energetically favourable for it to split into sub-rivulets. 


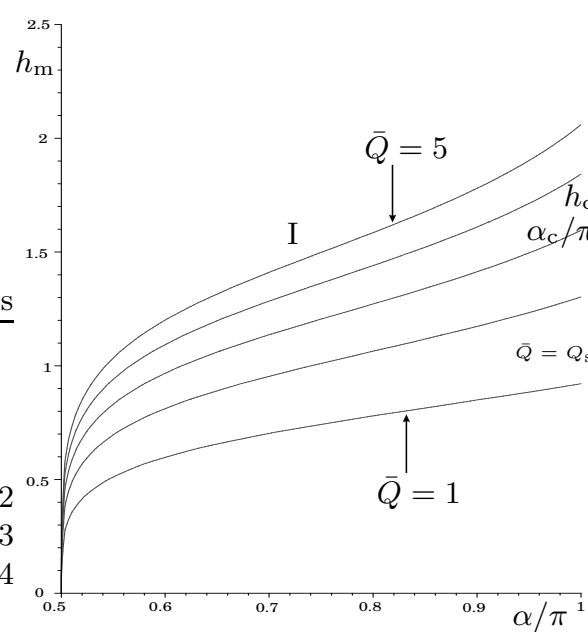

Fig. 3. Plot of $h_{\mathrm{m}}$ as a function of $\alpha / \pi$ when $\tau=1$ for $\bar{Q}=1, \ldots, 5$. Note that all solutions are of type I.

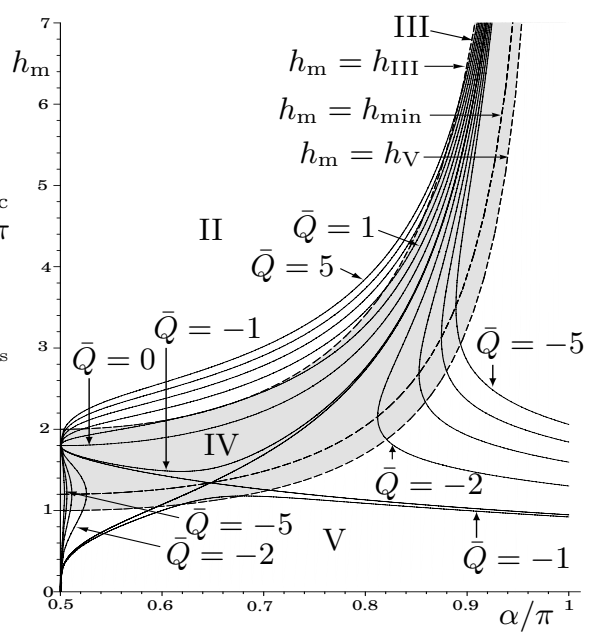

Fig. 4. Plot of $h_{\mathrm{m}}$ as a function of $\alpha / \pi$ when $\tau=-1$ for $\bar{Q}=-5, \ldots, 5$ showing when the different types of flow pattern occur.

\section{References}

1. S. V. Alekseenko, P. I. Geshev, and P. A. Kuibin "Free-boundary fluid flow on an inclined cylinder," Soviet Phys. Dokl. 42, 269-272 (1997).

2. A. C. King and E. O. Tuck, "Thin liquid layers supported by steady air-flow surface traction," J. Fluid Mech. 251, 709-718 (1993).

3. T. G. Myers, H. X. Liang, and B. Wetton, "The stability and flow of a rivulet driven by interfacial shear and gravity," Int. J. Non-Linear Mech. 39, 1239-1249 (2004).

4. A. Oron, S. H. Davis, and S. G. Bankoff, "Long-scale evolution of thin liquid films," Rev. Mod. Phys. 69, 931-980 (1997).

5. H. H. Saber and M. S. El-Genk, "On the breakup of a thin liquid film subject to interfacial shear," J. Fluid Mech. 500, 113-133 (2004).

6. M. Villegas-Díaz, H. Power and D. S. Riley, "On the stability of rimming flows to two-dimensional disturbances," Fluid Dyn. Res. 33, 141-172 (2003).

7. S. K. Wilson and B. R. Duffy, "A rivulet of perfectly wetting fluid draining steadily down a slowly varying substrate," IMA J. Appl. Math 70, 293-322 (2005).

8. S. K. Wilson and B. R. Duffy, "When is it energetically favorable for a rivulet of perfectly wetting fluid to split?" Phys. Fluids 17, 078104-1-07801-3 (2005).

9. S. K. Wilson and B. R. Duffy, "Unidirectional flow of a thin rivulet on a vertical substrate subject to a prescribed uniform shear stress at its free surface," Phys. Fluids 17, 108105-1-108105-4 (2005). 